\title{
RISK ASSESSMENT IN DEVELOPMENT OF LEAN ARCHITECTURE FOR CONTROL SYSTEM OF AERO ENGINE
}

\author{
Jha B K*, Ramachandra S, Srinivasa murthy P N \& Hans P \\ Gas Turbine Research Establishment, \\ Defence Research \& Development Organisation, \\ CV Raman Nagar, Bangalore, India \\ jhabk@gtre.drdo.in
}

\begin{abstract}
A modern gas turbine engine functions with a full authority digital engine control (FADEC) unit. This, in addition to providing superior controls, makes pilot free from the anxiety of monitoring engine performance and health. This confidence is a result of reliable machine (gas turbine) along with complementing control system. Many a times short comings of the individual aerodynamic modules ,structural issues, subsystem related concerns, matching of components, scaling of performance degradation over time, health management and performance management must be complimented by a smart control system. Hence it is imperative that control system has to be built with high reliability and redundancy to improve the reliability parameters. This very philosophy increases complexity of the system as well as increased part count and weight during development.
\end{abstract}

Interplay among many design parameters warrants Systems Engineering (SE) approach as an essential approach for effective management of complexity and change. SE process has to be used for concept exploration and design path formulation to reap the benefit at the later part of life cycle costs. Development of a complex system is recursive and conflicting during various life cycle stages. Projects undertaken in the previous century used to be more than 10-15 years duration and today similar projects are to deliver with reduced time frame. The main thrust is on automating and optimizing the processes, with continual improvement of quality and reducing waste. Management of change along with empowering the resources and manpower makes it indispensable use the lean principles to build these complex systems. Lean Architecture encompasses the above requirements.

An existing control system having cleared by IV\&V team and has matured to Technology Readiness Level (TRL) of 6-7 is considered in this study for upgradation for future application. A modern control system is being upgraded and lean architecture principle are followed to optimize the system along with reduction of weight by understanding the physical system design and data study from Integrated vehicle health monitoring (IVHM) system. The upgradation or redesigning approach can be inferred through various decision making process of systems engineering. This paper brings out the risk assessment concept of reducing the complexity through Analytic Hierarchy Process (AHP) approach where various alternatives, criterions and sub-criterions are evaluated and thereby assessing the risk in attempting to reduce the complexity and weight.

Key words: risk assessment, lean architecture, systems engineering, gas turbine, control system

*Corresponding Author: jhabk@gtre.drdo.in Ph: +91 80-25040414, Fax: +91-80-25241507 